\title{
A Meta-metamodel for Dynamic Constraint Feedback in Modeling Languages
}

\author{
Matt Bunting \\ University of Arizona \\ Tucson, Arizona, United States of America \\ mosfet@email.arizona.edu
}

\author{
Jonathan Sprinkle \\ University of Arizona \\ Tucson, Arizona, United States of America \\ sprinkjm@email.arizona.edu
}

\begin{abstract}
Complex design considerations of Cyber-Physical Systems (CPSs) are greatly aided through the use of high-level abstractions in a Domain-Specific Modeling Language (DSML). A DSML environment ensures that models will be correct by construction, however syntax correctness does not imply dynamic safety. Presented is an approach to define a meta-metamodel for DSML design with built in closed loop verification feedback for model correction based on dynamic constraint violations. Usage of this framework during DSML design can benefit CPSs such as autonomous vehicles by ensuring that designs are safe, through codified expert knowledge and verification tool integration. A case study is shown using a simple hybrid controller modeling language and reachability verification tools.
\end{abstract}

CCS Concepts - Software and its engineering $\rightarrow$ Domain specific languages; • Computer systems organization $\rightarrow$ Embedded and cyber-physical systems; • Hardware $\rightarrow$ Model checking.

Keywords Domain-Specific Modeling, Cyber-Physical Systems, Verification Tools

ACM Reference Format:

Matt Bunting and Jonathan Sprinkle. 2019. A Meta-metamodel for Dynamic Constraint Feedback in Modeling Languages. In Proceedings of the 17th ACM SIGPLAN International Workshop on DomainSpecific Modeling (DSM '19), October 20, 2019, Athens, Greece. ACM, New York, NY, USA, 9 pages. https://doi.org/10.1145/3358501.3361239

\section{Introduction}

Cyber-Physical System (CPS) design is difficult due to the multiple domain considerations of networking, computation, and control systems [2]. Managing such complexity is greatly

Permission to make digital or hard copies of all or part of this work for personal or classroom use is granted without fee provided that copies are not made or distributed for profit or commercial advantage and that copies bear this notice and the full citation on the first page. Copyrights for components of this work owned by others than ACM must be honored. Abstracting with credit is permitted. To copy otherwise, or republish, to post on servers or to redistribute to lists, requires prior specific permission and/or a fee. Request permissions from permissions@acm.org.

DSM '19, October 20, 2019, Athens, Greece

(c) 2019 Association for Computing Machinery.

ACM ISBN 978-1-4503-6984-8/19/10.

https://doi.org/10.1145/3358501.3361239 aided through use of high level abstractions, where lower level implementations are based on well known practices. A Domain-Specific Modeling Language (DSML) may be designed considering such abstractions, to formally define the syntax of model design. Using a metamodel in a DomainSpecific Modeling Environment (DSME), models will be ensured to be correct by construction through structural constraints [5] [10] [11]. A model may be correct through the perspective of syntax, but may not necessarily behave correctly and could potentially violate dynamic safety constraints. Models created in such an environment will still need to undergo a proper verification process to check that all requirements have been met [16].

In a typical DSML, dynamic constraints are not modeled since the constraints will not change how low level artifacts are generated. The modeling user is then responsible for ensuring that their model behaves correctly under a set of dynamic requirements. Artifacts may however be generated not only for a functional solution, but also for verification tools. The output of verification tools may then be compared against the set of requirements. Should any constraint violation exist, the user could be notified of the behavioral error to modify their model into a potentially correct revision.

Model correction may also be performed automatically. A designer of a DSML may be already aware of best known design practices based on particular constraint violations. The designer could capture this knowledge by designing an expert block that takes constraint violations as an input, and performs a formally defined model transformation. Such a transformation may modify a value or modify the model's structure. Not all systems have trivial dynamics, and thus more dynamic approaches are needed to handle complex dynamics.

Figure 1 is a visual aid to the concept of closing the loop on Dynamic Constraint Feedback (DCF). An initial model is first provided as $x$, which may be directly deployed or may be interpreted into artifacts necessary for verification tools. Execution of a verification tool provides the set of dynamic behaviors $b$, which may be compared against the set of requirements $r$. Should any constraint violations $e$ exist, an expert block may decide on the appropriate transformation method. With a known model modification method $u$, an update to the model may be performed. This cycle may be 


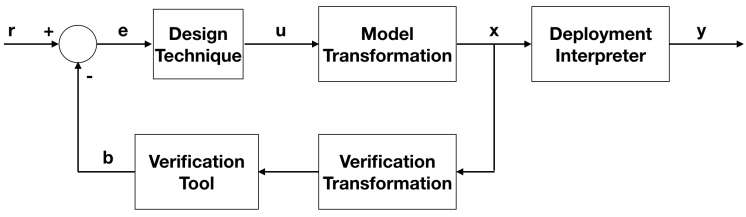

Figure 1. A closed-loop dynamic constraint feedback modeling language.

performed if necessary multiple times until all constraint violations are removed.

Design of a DSML with interpreters is already a challenging task, so designing a DSML with DCF adds even more development time. Our approach to aid in the development process is through the introduction of the Dynamic Constraint Feedback Modeling Language (DCFML). The intention of the DCFML is to reduce development time in DSMLs implementing DCF while increasing the utility of produced DSML's with the inclusion of DCF. This not only aids the DSML developers, but users of the language can see benefits by being provided with expert knowledge during the design of their model. Models produced will also be ensured to not violate any defined constraints, resulting safer CPS production.

Hybrid controller design is a common practice in the CPS field. A case study involving hybrid controller design is also discussed to show the potential application of the DCFML. The language design involves a brief overview of how a DSML can be realized using the described framework. Offthe-shelf verification tools of MATLAB's stepinfo and HyCreate are also implemented in this example.

This paper first discusses the background of DSMEs, model transformations, DCF, and hybrid verification tools in Section 2. Section 3 then introduces the DCFML and discusses the design of subcomponents. A case study involving hybrid controller design is then shown as an implementation of DCFML in Section 4. Finally, some of the limitations of the current stat of work and other concluding remarks are discussed in Section 5.

\section{Background}

\subsection{DSME}

A DSME provides the necessary tools to define the meta specification and to build models based on the formal specification [8]. The use of a DSME can greatly aid in the success of designing a CPS [9]. The Web-based Generic Modeling Environment (WebGME) [17] is an example DSME that provides a method of designing a metamodel that closely represents a class diagram from the Unified Modeling Language (UML). This particular environment is well suited for software experts with experience in UML and Javascript. Design of a language syntax is fairly easy due to model visualizations, enabling rapid prototyping of the formal specification. WebGME also features template generation for decorators, add-ons, and plugins. The Javascript based plugins using WebGME's Core API are invokable through the web interface and provide model traversal and modification methods. The Core API is the effective method of designing model interpreters and model transformations. Model interpreters provide an important utility for DSMLs by producing real working low level artifacts.

Model transformations encompass the design of functions that take an inputted set of source models and produce a set of target models. Source and target models may exist at different abstraction layers and with different or similar metamodel definitions [7] [12]. An interpreter or compiler for example transforms the model from a high level language into a low level artifacts [19]. Conversely, rewriting a program written in $\mathrm{C}++$ into Java would be at the same abstraction level but under a different formal specification [19] . Model transformations may also exist as model evolution by modifying the functional graph, maintaining the metamodel between the source and target models by only needing to modify the model structure.

Some frameworks have been written to handle a variety of transformation methods such as ATL for OMG [13] [12]. GReAT is an example that works with the Generic Modeling Environment, the prior version of WebGME [4]. Such transformation tools work directly with the metamodel definitions to easily provide methods to integrate models transformations in a DSME.

\subsection{Dynamic Constraint Feedback}

One of the natural efforts in designing a model interpreter is to run a model in a real system to check the system dynamics. This leads to evaluation of the system where the syntax alone cannot check that dynamic constraints have been met. Interpreters may also be written to translate the model into artifacts for verification and simulation tools for DCF. Past efforts have been conducted to use DCF in particular custom modeling languages [22] [21]. These works encompass real world engineering problems with custom made expert blocks to automatically correct the model. This includes controller tuning through value modification or adding new gains to an existing controller. Other efforts have implemented DCF using very different DSMLs, such as concurrent state diagram modeling using the SPIN model checker [23]. In this example, concurrent state machines issues such as deadlock are corrected by adding states or transitions.

Notable in these prior other works with DCF based model correction is that a high level perspective is used when describing the methodology, but each example's implementation is performed at a low level in the modeling language design. The focus in each work is on the methods used to 


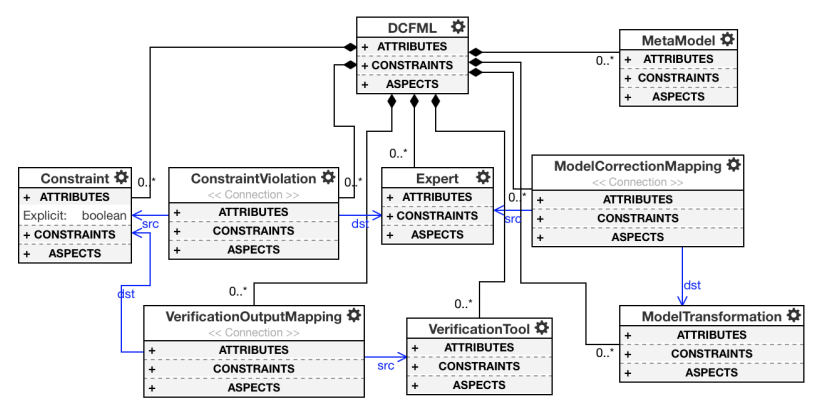

Figure 2. The meta-metamodel of a modeling language considering dynamic constraint feedback.

close the loop on model correction. Each describes the definitions of how models are created and should be modified based on the output of specific verification tools. The data types at each stage vary greatly, though the high level discussion about the design is very similar in each work. It is these past works that serve as one of the focuses for the design presented in this work.

\subsection{Hybrid Systems Verification}

Often in complex systems like CPSs, simple controllers are not sufficient to satisfy complex requirements. One method of making use of traditional control theory but attempting to satisfy important safety constraints is hybrid control design [15]. Hybrid controllers change their control modes based on particular conditions, thus combing state diagram design with control design. Different tools are available to simulate a hybrid control design but vary in their methods of evaluations, such examples are dReach [14] and HyCreate [3]. Due to the extensive research being done on reachability and its importance to CPSs, this serves as a great case study for the use of the DCFML. Design of hybrid controllers may even be reduced to simpler, easier to design modes yet still need to consider critical safety constraints [18] [6]. For simple controller design such as a Linear Time Invariant (LTI) system, some common practice heuristic based methods include the Ziegler-Nichols method [1]. Online resources are also being built to create a taxonomy of verification tools for easier discovery and tool selection [20]. Such online resources include examples for implementation methods, potentially in the future example modeling languages and metamodels could be provided for easier DSML integration.

\section{The Dynamic Constraint Feedback Metamodeling Language}

\subsection{Overview}

The DCFML has components that may be thought of as a functional mapping between different parts in a component based design. An overview of the framework may be built

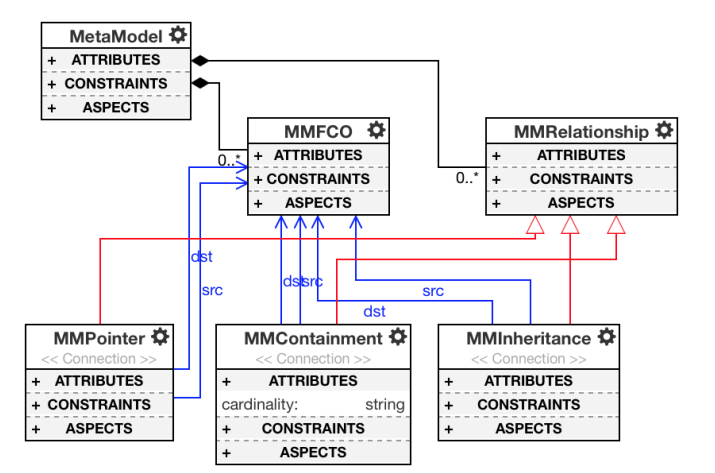

Figure 3. The formal specification of a metamodel, i.e. the meta-metamodel.

as a metamodel using WebGME following from the high level perspective in Figure 1. The metamodel for DCFML is shown in Figure 2. A main difference between the two figures are how edges are defined, where the metamodel needs to represent the connections as models representing a mapping. For example, the output of verification tools needs to be associated with corresponding dynamic constraints, so the VerificationOutputMapping model provides this functionality. Some other notable differences are the exclusion of the deployment interpreter and the comparator block. The comparator block is purely implied since it is not needed to create the block for each constraint. Instead, each constraint is mapped to an expert block, implying that a violation of the particular constraint will be provided to the expert. The lack of the deployment interpreter is due to not being a necessary part of the closed feedback loop.

\subsection{Metamodel}

The metamodel is the formal specification of how models are constructed and drives how model transformations are to be defined. Figure 3 shows the formal specification of a metamodel, i.e. the meta-metamodel. This meta-metamodel is designed to replicate how common DSMLs are constructed in WebGME. This meta-metamodel does not capture the full capabilities of metamodels, but is sufficient for the DCF framework in simple languages. First Class Objects (FCOs) are replicated with a similar name, the MetaModel FCO (MMFCO). This is also true for three class relationships for Inheritance, Containment, and Pointers. WebGME provides the ability to decorate objects in a model and even includes their own metamodel style decorator to make models produced under this meta-metamodel appear like a WebGME metamodel. 


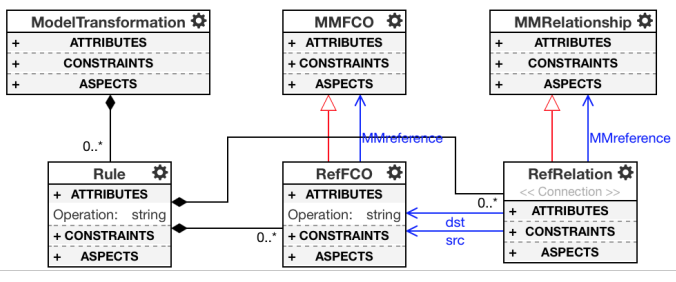

Figure 4. Model transformation definitions, relating to components in the metamodel.

\subsection{Constraints}

In the prior works that implemented a method of DCF, constraints were defined either implicitly by the design domain (i.e. controller stability, no deadlock) or they were defined explicitly (i.e. controller rise time, accepting states). Implicitly defined constraints do not need to be captured by the metamodel since they are assumed to be held for any model, but explicitly defined constraints vary between models and need to be defined. For DCFML, all constraints need to be defined for closing the loop. Figure 2 shows the method of constraint capture, with an attribute to define whether the constraint is explicit or implicit. The constraints also contain a set of datatypes to define structure of the constraint. Upon interpreting a model of a DSML from DCFML, explicit constraints become an addition to the metamodel which will be shown in the case study.

\subsection{Expert Block}

Each expert block in the feedback loop may require a complex set of rules and heuristics. Currently this expert block serves as a placeholder since modeling an entire set of possible algorithms into a single metamodel is outside of the scope of this work. The case study will only provide a simple reactive set of expert blocks which will encompass a simple mapping between violations and model transformations. More complex expert blocks may involve the coordination of multiple methodologies, and may need to include functionality like history tracking and stopping criteria. An example of this is the Ziegler-Nichols controller tuning method [1]. Here a model needs to be dynamically tested until a particular state of tuning is reached before proceeding with the next phase of tuning involving different gains to be adjusted.

\subsection{Model Transformations}

For DCFML, model transformations serve as a method to evolve a model into a more correct solution. An already available transformation language could also potentially be used in place of the transformation definitions defined in this DCFML. GReAT would be a good fit, but support is only available for GME instead of WebGME. Since only model evolution is needed for the DCFML, transformations needed are endogenous as both the source and target models are from the same metamodel. This is further simplified by limiting the types of transformations to either adding, deleting, or modifying attributes of model components. There are perhaps more evolution techniques needed when considering general domains, but these simple rules are sufficient for the case study.

Figure 4 shows the metamodel for transformations. This metamodel is inspired by simple examples in GReAT. Each transformation method has a set of rules, where rules contain references to the metamodel. Each reference to a metamodel component also has an attribute that defines the function of the reference. These functions include either adding, removing, changing an attribute, or no action.

Interpretation of the model transformation definitions provides source code in the form of functions with inputs relating the the references. These functions require the WebGME identifier of the blocks, a necessary part to ensure that the correct blocks are added and related to existing instances in the model. Each expert block is responsible for deciding which transform needs to be performed.

\section{Simple Hybrid Controller Modeling Language using DCFML}

\subsection{Overview}

A simple case study may now be constructed using the meta definitions described in the DCFML definition section. We start by defining the design domain in a concise manner to ease design complexity. This example DSML is based on controlling an autonomous, Ackerman steering style vehicle using hybrid control techniques to reach a particular goal. Gains for the each control mode may be modified, and the time that a controller is active before transitioning to a sequential mode is also settable. Figure 5 shows the model of DCF constructed using the metamodel from Figure 2. A decorator is used in the model builder to help distinguish between different DCF components. This DCF model incorporates two different verification tools, three constraints, two experts, and two transformation techniques. The metamodel appears disconnected, however it contains the meta specification needed to define the transformations.

\subsection{Metamodel}

Inside of the HybridMetaModel block the metamodel is constructed. Figure 6 shows the metamodel definition. This not an all-encompassing method to model all hybrid controllers, but is rather targeted for the simple domain of driving a car to a particular location. An example of a controller a user may construct using this metamodel is in Figure 7. Each mode transitions to another after a defined amount of time in seconds resulting in modes acting sequentially. Each mode has attributes corresponding to the controller set points for 


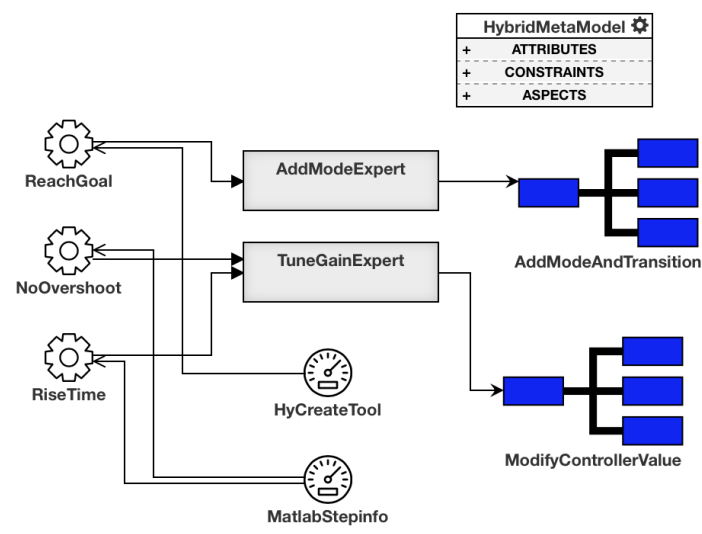

Figure 5. The DCF design of a simple hybrid controller language

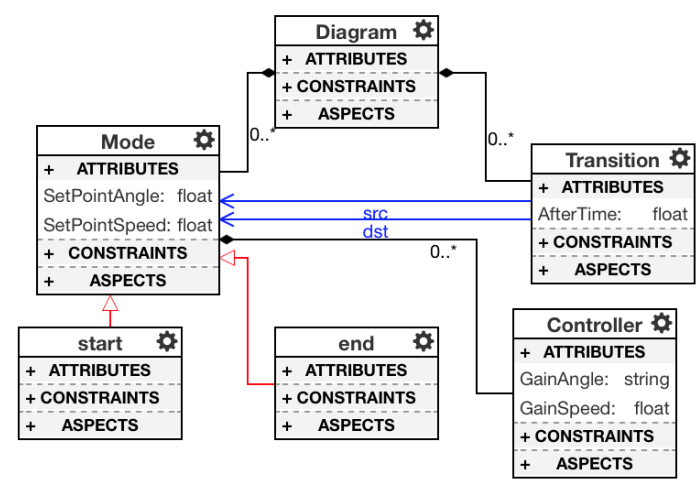

Figure 6. The metamodel of a simple hybrid controller.

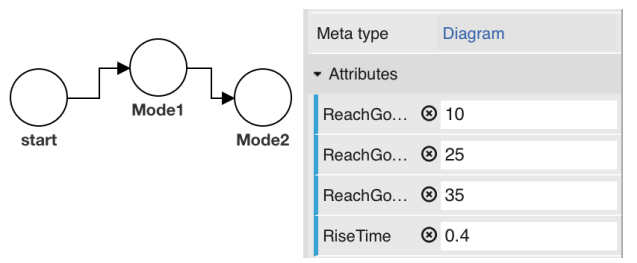

Figure 7. An example set of modes in a Diagram constructed using the meta in Figure 6. Also shown is the inclusion of explicit constraints that are user definable.

both speed and tire angle. Modes also contain a control law to set gains for steering and speed.

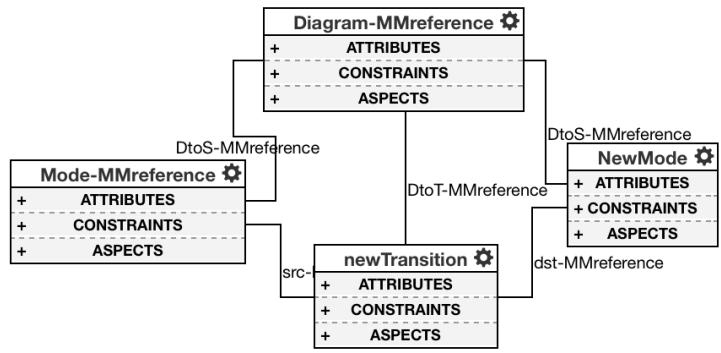

Figure 8. A rule in the AddModeAndTransition model transformation using the HybridMetaModel in Figure 6.

\subsection{Model Transformations}

The example language features two model transformation examples: AddModeAndTransition and ModifyControllerValue. Figure 8 shows a rule inside the AddModeAndTransition transformation. This transformation is responsible for adding a new mode, then connect a new transition from an existing state to the new state. The new state and transition need to be referenced to both an already existing state, and the parent diagram. Here there are two components that are needed for reference, the original diagram and a state where a new transition is to be connected.

Appendix A shows the code generated from interpretation of the transformation in Figure 8. The function shows that the references in the transformation meta result as function inputs, a necessary part to place models under the right parent and to build appropriate associations. Figure 9 demonstrates a simple execution of the model transformation on a set of sequential modes. The reference mode is the final mode in the sequence, and is highlighted to show WebGME's ID which is used for correct placement when invoking the function shown in appendix A. After transformation execution a new mode is created along with a new outgoing transition form the referenced mode.

\subsection{LTI Verification}

Constraint modeling is based on the capabilities of the verification methods due to the need for tool outputs to be directly compared. The constraints of the system will be to ensure that a system will be able to reach a particular goal starting from the origin, and make sure that there is no overshoot in each controller while being under a particular rise time. Three different verification tools are explored for this language. MATLAB's stepinfo is a simple function to execute and provides simple results that may be directly comparable to primitive constraint data types. For this example language we may be able to assume that a 2 nd order model was validated to represent the plant $P$ of the car in the $s$-domain as the following:

$$
P=\frac{1}{s^{2}+20 s+40}
$$




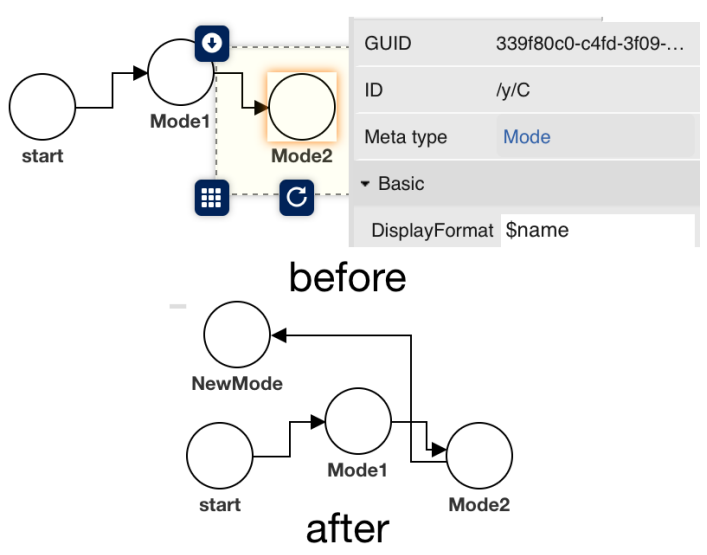

Figure 9. An example model before and after the AddModeAndTransform is executed. In the before image, the state of interest is highlighted to show mode of interest, representing the NewMode in Figure 8.

This model of the car is not something that the user can change since this is a language specifically designed for a particular system. Our controller may then have a proportional gain set by the user at an initial value of 100 . An interpreter would then need to be designed to take the prior knowledge of the system plant, along with user set controller design. The following script is an example of generated MATLAB code from the stepinfo verification interpreter.

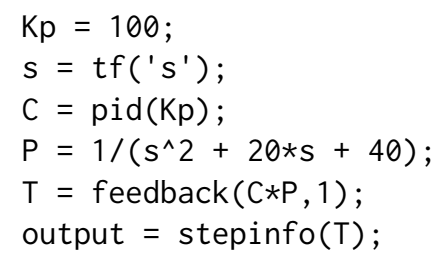

Executing this script results in following output:

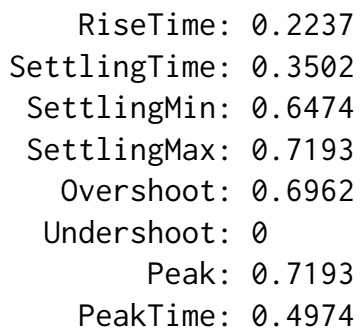

This is a trivial verification tool to implement, especially if the plant is not needed to be modeled; however it provides directly comparable information against the constraints of rise time and overshoot. One note is that this is only for one particular mode, whereas a hybrid system is usually intended to be built with multiple control modes, thus implying that this tool needs to be run against each mode. This tool is sufficient for checking two of the three constraints, however it cannot handle running the constraint of reachability.

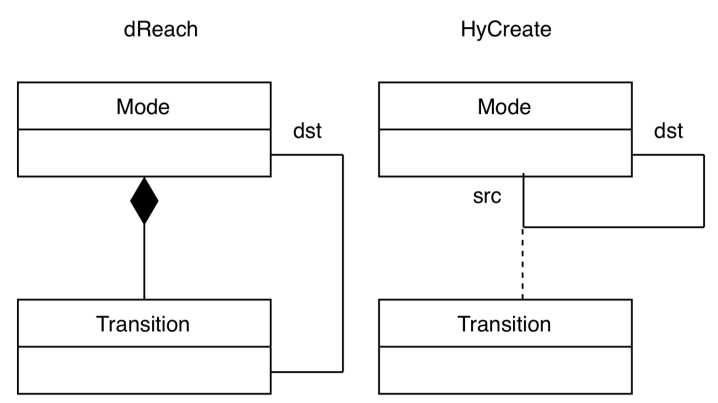

Figure 10. Configuration file abstraction for dReach and HyCreate hybrid system models.

\subsection{Reachability Verification}

Two different tools were inspected for checking a controller's reachability. dReach [14] and HyCreate [3] provide different methods of computing reachability using a hybrid system. Either of these tool will work for the purposed of this simple language, but there are scenarios where some of the methods of computation and features in each tool may be useful in particular domains. For example, dReach can solve for the specific initial conditions necessary to achieve a strict reachable state.

Both of these tool are based on hybrid control system design, allowing for the modeling of modes and transitions. To generate files for each tool, configuration files can be reverse-engineered for their internal structure. When abstracted, each of these languages model hybrid systems using a different metamodel. Figure 10 shows the corresponding class diagrams of each tool. In dReach, outgoing transitions are contained within each mode, whereas transitions are separate entities that manage an association between modes in HyCreate. Note that both of these metamodels also differ from the standard of WebGME metamodel design, where connective model is associated through pointers. All of these different modeling techniques are certainly valid for modeling a hybrid system, but demonstrates the need for model transformation methods to map a model from one metamodel to a different design paradigm.

Each tool also has very different lower level syntax. HyCreate files are written in XML format while dReach has its own custom yet easily readable syntax. dReach is mostly order agnostic by referring to different modes through a unique integer. HyCreate requires a strict ordering, so each mode needs to have derivatives defined in the same order. HyCreate functions by generating Java code, then compiles and runs the simulation to provide reachability data. Due to this, derivatives and guards needs to be written in a particular syntax. This can complicate interpreter writing, for example to call a sinusoidal function in dReach, using $\sin (\mathrm{x})$ would suffice whereas HyCreate would use Math.sin(x). 
As an example of the complications, in dReach, a derivative is written as follows

$\mathrm{d} / \mathrm{dt}[\mathrm{v}]=((((r-v) * 1) / 1)-((v * 0.2) / 1))$;

whereas in HyCreate, the same derivative is written as

return new Interval $(((((\$ R-\$ V) * 1) / 1)-((\$ V * 0.2) / 1))$, $((((\$ R-\$ V) * 1) / 1)-((\$ V * 0.2) / 1)))$;

Handling the syntax in one particular language is not very difficult, but generalizing a method for all tools is a challenging problem. This is especially true when defining specific properties in one tool that have similar or no use in another. A single interpreter has been written to generate code for both tools described. Normally an interpreter can directly translate models into artifacts, however here a model is first translated into a data model in the JavaScript Object Notation (JSON) format. The structure of the data model closely represents the metamodel, but has added attributes for easier model traversal. For example, a mode under the specification of WebGME or HyCreate has no information on the incoming and outgoing transitions, which would be a helpful part of generating a dReach configuration file. Likewise, only modes have transitions under the dReach model, so iterating over just transitions for HyCreate file generationg is a challenge. The data model has all components flattened for easy iteration, and each component has references to all connected components. Each mode has a list of incoming and outgoing transitions, and transitions are contained under the diagram with references to the source and destination.

With this data model as an intermediary step, the artifact generation becomes fairly trivial for both tools. The data model is also populated with elements that are tool specific, such as the faceSizeRatio and GridSize needed for HyCreate, and the unique integers for each mode needed for dReach. Math expressions for derivatives are also generated for inclusion in the data model. Embedded javascript may then be used to fill out a template version of a configuration file for each tool. A data model snippet of a derivative in a mode is shown in JSON format where differences in expressions and particular tool specific attributes are shown:

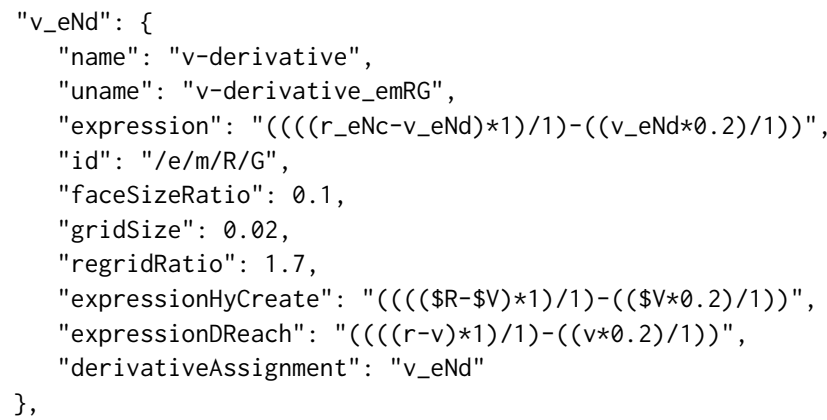

The practice of transforming a model to fit two different verification tools shows how further verification tools could be more quickly implemented. Production of a flat data model could be in a generalized form for a majority of metamodels. Verification tools would then only need to
Table 1. Gain tuning from overshoot violation

\begin{tabular}{ccc}
\hline Iteration & Gain & Overshoot \\
\hline 1 & 100 & 0.6962 \\
2 & 90 & 0.3228 \\
3 & 81 & 0.1054 \\
4 & 72.9 & 0.0127 \\
5 & 65.61 & 0 \\
\hline
\end{tabular}

Table 2. Gain tuning from rise time violation

\begin{tabular}{ccc}
\hline Iteration & Gain & RiseTime \\
\hline 1 & 10 & 0.7693 \\
2 & 14 & 0.7045 \\
3 & 19.6 & 0.6284 \\
4 & 38.416 & 0.4531 \\
5 & 53.7824 & 0.3636 \\
\hline
\end{tabular}

be a skeleton version. For the purposes of this particular hybrid design language, only one verification tool is needed to assess reachability. HyCreate was chosen based purely on ease of execution, though switching to dReach in the future will be easier for backend server integration.

\subsection{Expert Blocks for LTI}

There are two designed expert blocks for the simple hybrid language to handle the three possible constraint violations. The first two constraint violations of rise time and overshoot are handled by the same expert block involving controller tuning. The controller tuner is provided with constraint violations and then makes adjustments to the corresponding controller gains. As an example, a user may define the rise time constraint to be 0.4 seconds but must never tune the gains too high to create overshoot. From the previous example, we see that small amount of overshoot does exist, resulting in a constraint violation. We also see that the rise time has not exceeded the user's defined constraint. A heuristic based expert block may then have a rule to decrease the gain by $10 \%$, then try again. Using this rule, Table 1 shows how the dynamics of the model evolve over each iteration.

This heuristic appears to work well for overshoot violations. Likewise, if the gain is tuned to be too small, then the rise time will be too slow. If we start with an initial model with a gain of 10 , then the rise time will be 0.7693 . Similar to the overshoot handling, the expert may be designed to increase the gain by $40 \%$ until the rise time does not violate the constraint. This rule results in the iterations shown in Table 2. 


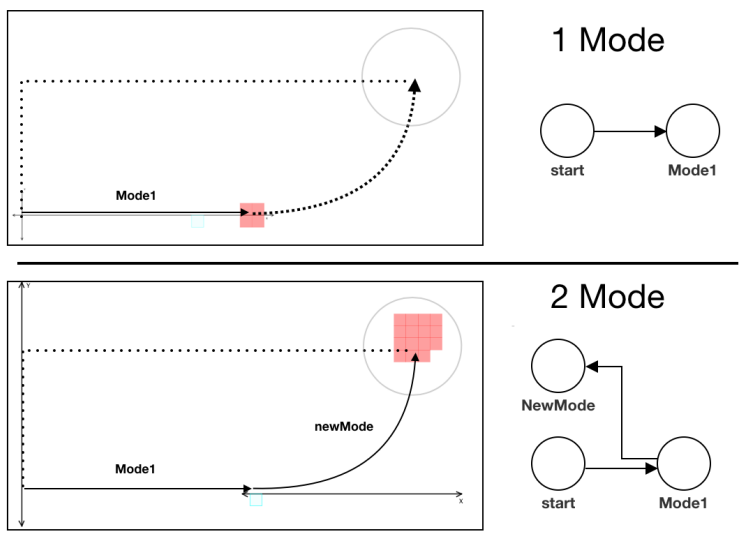

Figure 11. Output of HyCreate with a single mode system, marked to show the unreached circle. A second system is shown after model transformation with the necessary added mode to reach the goal coordinates.

\subsection{Expert Block for Reachability}

The expert block for the constraint of reaching a particular set of coordinates within a radius is based on the reachability information provided by HyCreate. A final reachable set that is not within the target circle needs to have a modification that could occur either with a change in gain tuning, a transition time, or an additional mode. This expert takes the approach of adding a control mode at the end of the control sequence. Figure 11 shows a simple example illustrated on data provided from HyCreate. The first model iteration only has a single control mode, driving the vehicle along the $\mathrm{x}$-axis. This does not achieve the target circle. The expert block in this case takes the centroid of the final reachable set and solves for the tangent circular path needed to reach the goal center. The path information is then used to set the controller set points in a new mode. Invocation of the model transformation shown in figure 8 then modified the model, shown as 2 mode solution. This the new mode, the HyCreate tool is invoked once more and demonstrates a proper reachable set.

For this particular language, only simple solutions to the expert blocks were introduced. Though this simple case can be solved by this expert, it is clear that the reachable set became larger in area. For a more complicated system with many modes, this may grow large enough to break feasibility of this solution. Instead a reduction in modes and gain tweaking may be a better approach. This solution is here to illustrate a simple method of model evolution to correct a model.

\section{Conclusions}

The DCFML provides an extension to WebGME's metametamodel to consider DCF integration in DSML design. Implementing automatic verification methods in CPS based DSMLs can be a critical part to ensuring that models abide by important safety constraints. The verification loop can also potentially be closed to automatically correct constraint violations through model evolution. This framework aids a DSML designer by extending an already familiar metamodel with the methods needed for model transformation, constraint modeling, and expert block connectivity. By generating some of the necessary plugins needed for DCF, a DSML does not need to focus on some of the lower level implementation of model correction. Implementation of DCF is not a new idea, but the abstraction as an extension to the metamodel is new.

This work briefly looked at a case study implementing the framework into a simple hybrid controller designer. The hybrid controller DSML demonstrated the design of the metamodel, model transformation, expert blocks, and verification tool integration. Interpretation of the design automatically fit constraints into the metamodel, and generated code for model transformations. Expert blocks were also constructed to demonstrate simple methods of closing the loop. These are primitive examples but demonstrations were shown on how models can be automatically evolved into working solutions.

\subsection{Future Work}

The DCFML described is in its infancy, and parts of the design could be further defined. The expert block is lacking in its current state, requiring code to be written at a low level to be properly implemented. As discussed, expert blocks can vary greatly so abstraction for general purpose modeling is not a small task. Doing so could result in coupling model transformations with the expert block during system interpretation, greatly reducing the time of implementing DCFML.

Verification tools also vary greatly by their necessary inputs and outputs. Generation of a model interpreter to create a flat metamodel could greatly reduce implementation time. This way a DSML designer would only need to focus on building a template of verification tools rather than build custom transformations to produce verification tool artifacts.

\section{Generated Transformation Code}

The following is the function generated from the AddModeAndTransformation transformation in figure 8. This code is intended to be implemented in a Javascript-based plugin in WebGME. The Core API is responsible for performing the actual modification. The transformation model is used to determine the inputs for this function, in this case being references by WebGME generated IDs.

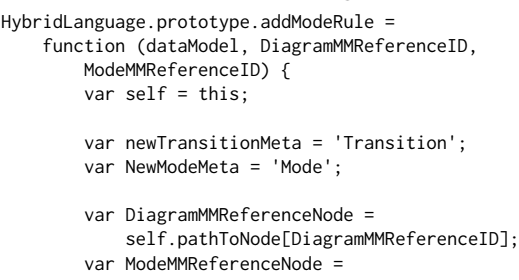




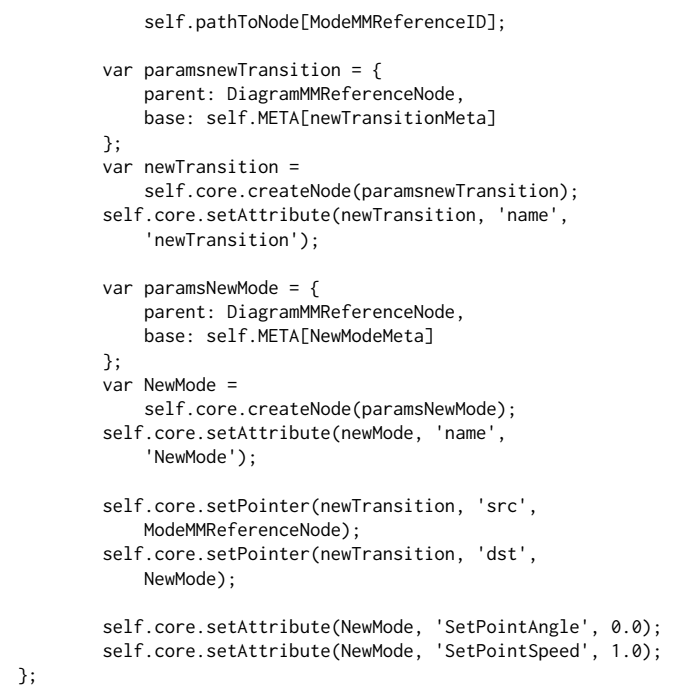

Modifying a model's structure is much more difficult compared to modifying attributes. The following function is a demonstration of modifying a node's value based on the node id, attribute name, and attribute value.

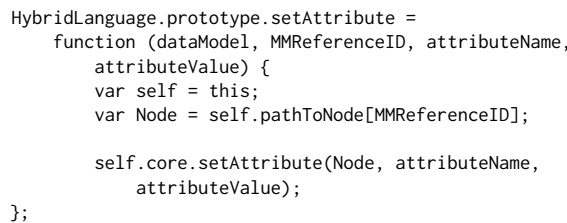

\section{Acknowledgments}

Support for this project was provided by the National Science Foundation under award 1253334. The authors would also like to thank the CPS Virtual Organization, award 0931632, for providing a verification tool resource for fast tool evaluation.

\section{References}

[1] Karl Johan Åström and Tore Hägglund. 2004. Revisiting the ZieglerNichols step response method for PID control. fournal of process control 14, 6 (2004), 635-650.

[2] Radhakisan Baheti and Helen Gill. 2011. Cyber-physical systems. The impact of control technology 12, 1 (2011), 161-166.

[3] Stanley Bak. 2013. HyCreate: A tool for overapproximating reachability of hybrid automata. Retrieved fanuary 17 (2013), 2016.

[4] Daniel Balasubramanian, Anantha Narayanan, Christopher van Buskirk, and Gabor Karsai. 2007. The graph rewriting and transformation language: GReAT. Electronic Communications of the EASST 1 (2007).

[5] Franck Barbier, Pierre Castéran, Eric Cariou, and Olivier Le Goaer. 2013. Adaptive software based on correct-by-construction metamodels. In Progressions and Innovations in Model-Driven Software Engineering. IGI Global, 308-325.

[6] Matt Bunting, Yegeta Zeleke, Kennon McKeever, and Jonathan Sprinkle. 2016. A safe autonomous vehicle trajectory domain specific modeling language for non-expert development. In Proceedings of the International Workshop on Domain-Specific Modeling. ACM, 42-48.

[7] Krzysztof Czarnecki and Simon Helsen. 2003. Classification of model transformation approaches. In Proceedings of the 2nd OOPSLA Workshop on Generative Techniques in the Context of the Model Driven Architecture, Vol. 45. USA, 1-17.

[8] James R. Davis. 2002. Model Integrated Computing : A Framework for Creating Domain Specific Design Environments.

[9] Patricia Derler, Edward A Lee, and Alberto Sangiovanni Vincentelli. 2012. Modeling cyber-physical systems. Proc. IEEE 100, 1 (2012), $13-28$.

[10] Mounira Kezadri Hamiaz, Marc Pantel, Benoît Combemale, and Xavier Thirioux. 2014. Correct-by-construction model composition: Application to the invasive software composition method. arXiv preprint arXiv:1404.0853 (2014).

[11] Mounira Kezadri Hamiaz, Marc Pantel, Xavier Thirioux, and Benoit Combemale. 2016. Correct-by-construction model driven engineering composition operators. Formal Aspects of Computing 28, 3 (2016), $409-440$

[12] Frédéric Jouault, Freddy Allilaire, Jean Bézivin, and Ivan Kurtev. 2008. ATL: A model transformation tool. Science of computer programming 72, 1-2 (2008), 31-39.

[13] Frédéric Jouault, Freddy Allilaire, Jean Bézivin, Ivan Kurtev, and Patrick Valduriez. 2006. ATL: a QVT-like transformation language. In Companion to the 21st ACM SIGPLAN symposium on Object-oriented programming systems, languages, and applications. ACM, 719-720.

[14] Soonho Kong, Sicun Gao, Wei Chen, and Edmund Clarke. 2015. dReach: $\delta$-reachability analysis for hybrid systems. In International Conference on TOOLS and Algorithms for the Construction and Analysis of Systems. Springer, 200-205.

[15] John Lygeros, Claire Tomlin, and Shankar Sastry. 1999. Controllers for reachability specifications for hybrid systems. Automatica 35,3 (1999), 349-370.

[16] Charles M Macal. 2005. Model verification and validation. In Workshop on" Threat Anticipation: Social Science Methods and Models.

[17] Miklós Maróti, Róbert Kereskényi, Tamás Kecskés, Péter Völgyesi, and Akos Lédeczi. 2014. Online Collaborative Environment for Designing Complex Computational Systems.. In ICCS. 2432-2441.

[18] Kennon McKeever, Yegeta Zeleke, Matt Bunting, and Jonathan Sprinkle. 2015. Experience report: constraint-based modeling of autonomous vehicle trajectories. In Proceedings of the Workshop on Domain-Specific Modeling. ACM, 17-22.

[19] Tom Mens and Pieter Van Gorp. 2006. A taxonomy of model transformation. Electronic Notes in Theoretical Computer Science 152 (2006), 125-142.

[20] Cyber-Physical Systems Virtual Organization. 2019. Verification Tool Library. https://cps-vo.org/group/verification_tools

[21] Sean Whitsitt. 2014. A Methodology for Mending Dynamic Constraint Violations in Cyber Physical Systems By Generating Model Transformations. (2014).

[22] Sean Whitsitt, Jonathan Sprinkle, and Roman Lysecky. 2014. Generating model transformations for mending dynamic constraint violations in cyber physical systems. In Proceedings of the 14th Workshop on Domain-Specific Modeling. ACM, 35-40.

[23] Kun Zhang and Jonathan Sprinkle. 2014. A closed-loop model-based design approach based on automatic verification and transformation. In Proceedings of the 14th Workshop on Domain-Specific Modeling. ACM, 1-6. 